SYPHILIS

\title{
Incorporating a social networking approach to enhance contact tracing in a heterosexual outbreak of syphilis
}

\author{
G Ogilvie, L Knowles, E Wong, D Taylor, J Tigchelaar, C Brunt, L James, J Maginley, \\ H Jones, M L Rekart
}

Objective: This report describes the outcomes of a social networking approach (SNA) in a heterosexually transmitted syphilis epidemic by street nurses in British Columbia, Canada.

Methods: Street nurses used SNA interviewing cues, environmental observation, peer outreach, serial interviewing, conducted blood tests, and offered treatment on the street. Rates of cases of syphilis identified by outreach nurses and cases linked to another case were compared before and after adoption of an SNA.

Results: SNA resulted in a significantly increased proportion of cases identified by the street nurses $(p=0.01)$ and increased the percentage of cases linked to a previous case $(\mathrm{p}=0.03)$.

Conclusion: This preliminary study confirms that SNA can increase the number of cases and contacts identified in an epidemic of a sexually transmitted disease in a vulnerable, hard to reach population.

S ince July 1997, the province of British Columbia, Canada, has had a sustained epidemic of infectious syphilis, as in many other jurisdictions worldwide. The provincial syphilis rate has increased from less than 0.5/ 100000 in 1996 to 4.5/100 000 in 2002. However, unlike many other international settings where the outbreak has been in the MSM (men who have sex with men) population, ${ }^{12}$ the outbreak in British Columbia has been primarily heterosexually transmitted, concentrated in the downtown eastside of Vancouver, in illicit drug users, sex trade workers (STW), and their patrons. The epidemiology of this outbreak, as well as a report on targeted mass treatment with azithromycin have been published elsewhere. ${ }^{4}$

Traditional control measures for sexually transmitted diseases such as focused contact tracing proved to be particularly difficult to employ in the affected population. Many STW were reluctant to name contacts as a result of fear of violence, codes of silence, and the genuine inability to remember names of contacts. Often, there was involvement with illicit drugs, as users, sellers, or both, creating additional barriers for naming partners. To improve our efforts to contain this outbreak, a social networking approach (SNA) was adopted. SNA is based on the concept that members of a core group and their sexual and social behaviours are critical to both the endemicity of sexually transmitted infections in geographic regions, and to their spread outside of these areas. ${ }^{5}$ A network informed approach for sexually transmitted disease control broadens the field of contacts to include a wider range of individuals including close friends, room-mates, and non-current sexual partners, among others. Non-traditional methods of contact tracing are employed to identify these individuals, and social networks are constructed. Once the networks are described, interventions such as education, testing, treatment, and follow up can be focused on individuals in the network, eventually impacting rates of disease transmission in the community. SNA has been advocated as an important component in the control and management of syphilis and other communicable diseases. This paper describes how the street nurse programme of the British Columbia Centre for Disease Control (BCCDC) incorporated an SNA to improve contact tracing and case finding in a heterosexual outbreak of infectious syphilis, and the outcomes of this approach.

\section{METHODS}

The street nurse programme of the Division of STD/AIDS Control at BCCDC has provided STD/HIV and harm reduction services to difficult to access clients in non-traditional settings such as shelters, transition houses, and in the alleys of the downtown eastside of Vancouver since 1988. To develop skills for identifying social networks, the street nurses attended the US Centers for Disease Control course "Fundamentals of STD Intervention" and in-services with experts in SNA. Injection drug user and sex trade peer outreach workers (PW) were hired to assist with accessing difficult to reach clients. A syphilis outbreak working group met weekly to discuss cases, identify potential contacts, and provide guidance on management. The street nurse outreach carry bag was adapted to include graphic syphilis educational pictures, a wider variety of condoms, and crack pipe mouth pieces, in addition to mobile phones, and devices to test for syphilis, such as needles and blood collection tubes.

\section{Contact tracing methods}

Before March 2002, traditional contact tracing methods were employed (table 1). This involved interviewing index cases of syphilis, and eliciting the names of sexual partners during the infectious period. Follow up of these partners ensued, and contacts would be offered testing, treatment, and contact tracing of their own partners. These methods were enhanced by SNA in March 2002. Street nurses conducted health interviews on the street, and SNA interviewing cues (location, event, partner lists) were incorporated to prompt cases and increase recall of sexual partners and to determine social networks. ${ }^{6}$ The question "Who do you think should be tested for syphilis?" was used to identify sexual partners of cases and other high risk individuals needing testing. Information from cases was gained over a series of encounters, rather than at a single interview. A checklist of places and contacts was developed for individuals with no fixed address, to find them when results were available. Environmental

Abbreviations: BCCDC, British Columbia Centre for Disease Control; MSM, men who have sex with men; PW, peer outreach workers; SNA, social networking approach; STW, sex trade workers 
observation at places of social aggregation for STW and drug use identified additional contacts. ${ }^{7} \mathrm{PW}$ helped access "hard core" individuals and define opportune moments for health care interviews and interventions. Street nurses used street language and strongly recommended syphilis tests, and conducted these tests and provided treatment on the street. Weekly SNA reviews permitted rapid change to accommodate developments in the outbreak.

\section{Data storage and analysis}

Before 2002, name, age, cultural background, and sexual partners were collected for each case (table 1). Data were stored on an Excel spreadsheet, and linkages with other cases was done manually. After introducing social networking, health data gathered by street nurses and PW were collected on a uniform data entry sheet. Information including appearance, location of contact, cultural background, distinguishing characteristics, participation in the sex trade, drug use, sexual and social contacts was entered into Epi-Data. Nurses could use this database to search for contacts of cases. Relevant fields from the Epi-Data database were imported into Epi-Info 6 then scripts were run to format the data so that the data could be used to draw social network maps, first in Pajek and then in Microsoft PowerPoint (fig 1).

Social network maps were drawn and reviewed by the street nurses. Street nurses also used social network maps to rapidly identify, contact, and test case linked individuals, often before contact with the case. Physicians communicated positive syphilis results immediately to street nurses, to facilitate rapid treatment of infected individuals.

Data for this analysis were obtained from the centralised provincial surveillance agency, the BCCDC. All positive syphilis tests in the province are reported to this agency. As

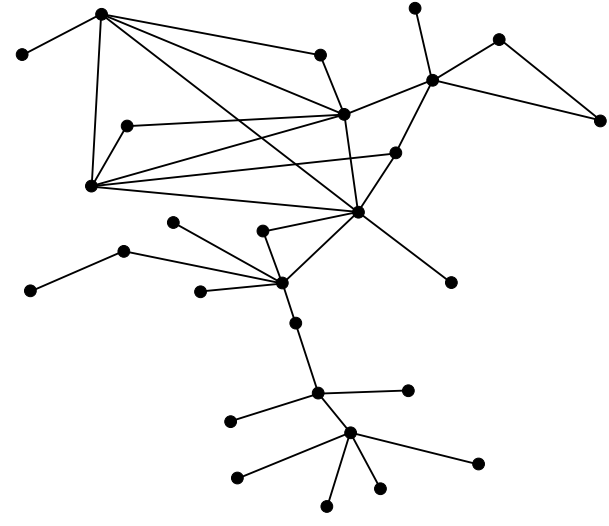

Figure 1 Syphilis social network map. Example of a social network map drawn in Pajek from contact information obtained through social networking. If a case of syphilis were identified in this map, any individuals linked to the case would be contacted by street nurses and offered education, testing and, if symptomatic, treatment. Street nurses would have blood testing equipment, educational materials, and treatment in their carry bag at all times, in order to offer these services at point of care. Location of street involved contacts could be identified through searching Epi-Data for information on usual hangouts, or through assistance of the peer outreach worker.

the main outcome of interest in contact tracing is identifying (and then treating) new cases of the infection, cases of syphilis identified by the street nurses and cases linked to another case were selected as the primary end points. The clinician who identified each case of syphilis was defined as the individual who ordered the syphilis test. If this clinician

Table 1 Contact tracing and data management and follow up before and after a social networking approach (SNA)

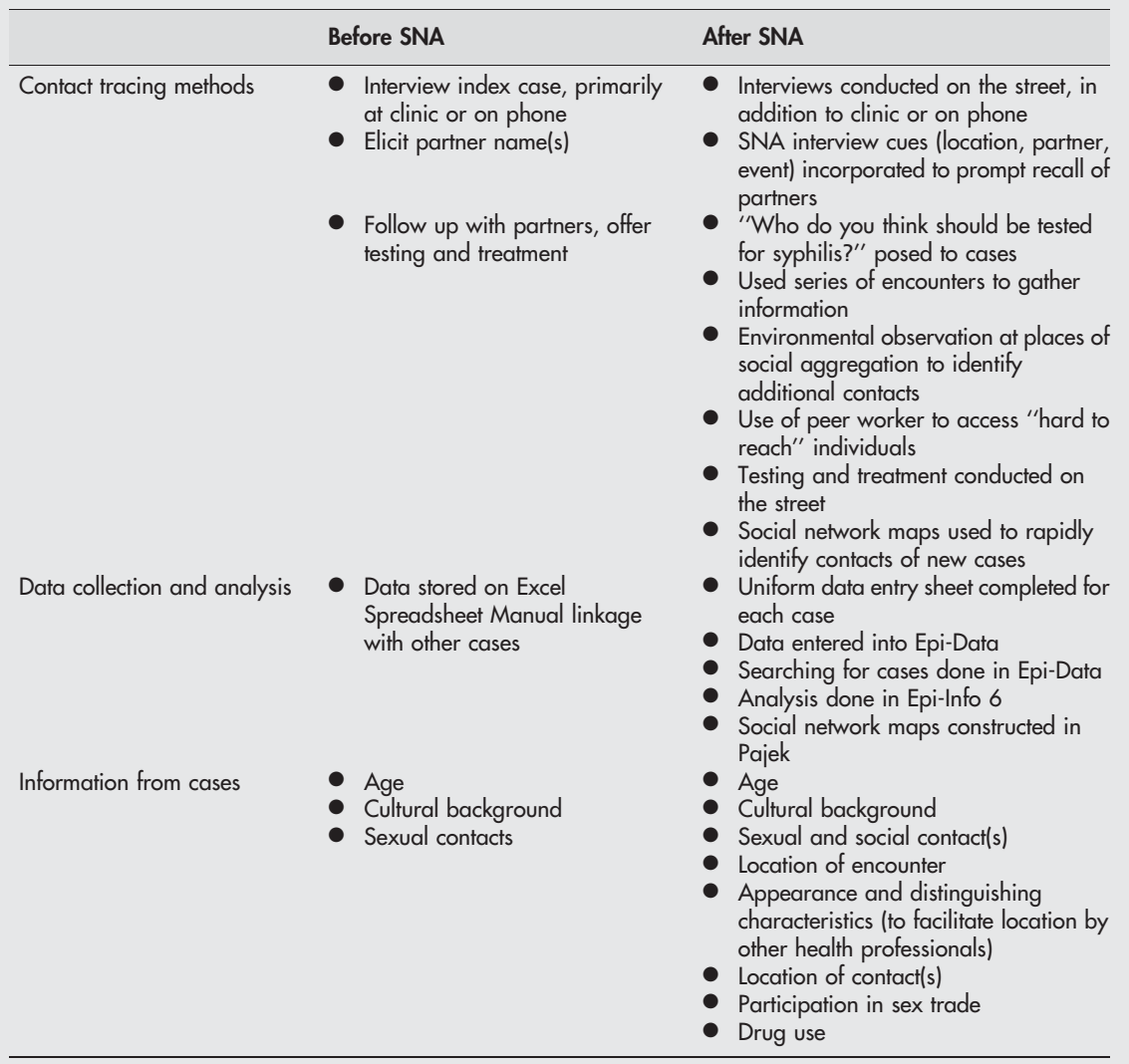


Table 2 Outcomes of social networking approach for syphilis epidemic

\begin{tabular}{lllll}
\hline & Before SNA (\%) & After SNA(\%) & OR (95\% CI) \\
\hline Case linked with another & $60 / 249(24.1)$ & $104 / 321(32.1)$ & $0.66(0.45$ to 0.96$)$ \\
Case identified by street nurse & $37 / 249(14.9)$ & $74 / 321(23.1)$ & $0.58(0.38$ to 0.90$)$ \\
\hline
\end{tabular}

was a street nurse, the case was defined as being identified by the street nurse programme. When a contact identified by a case tested positive for syphilis, this contact was defined as a linked case. Rates for both end points (cases identified by street nurses and linked cases) were compared for the 18 months before the introduction of SNA (March 2002) and after the introduction of SNA by Pearson $\chi^{2}$, with a p value of less than 0.05 being taken as significant. Data were input and analysed in Epi-Info 6, and maps were drawn in Pajek. Ethical approval for the study was obtained from the University of British Columbia (C03-0467).

\section{RESULTS}

Between 1 October 2000 and 31 March 2002, 249 cases of infectious syphilis were identified in British Columbia. Between I April 2002 and 30 September 2003, 321 cases were identified. In both time periods, over $60 \%$ of cases were identified in city of Vancouver, where the street nurses work (61.6\% $v 69.2 \% \mathrm{p}>0.05)$. Using an SNA, the percentage of cases of syphilis linked to a previous case increased by $8 \%$ $(p=0.03)$ and the overall percentage of syphilis cases in the province identified by the street nurse programme also increased from $14.9 \%$ to $23.1 \%(\mathrm{p}=0.01)$ (table 2 ).

\section{DISCUSSION}

In this evaluation, a street nursing programme that adopted a social networking approach increased both the percentage of linked cases and the overall percentage of cases of syphilis identified by the programme significantly. Adoption of a social networking approach was the main difference in the approach to contact tracing before and after March 2002. Elements of social networking that were likely to have contributed to these findings include enhanced contact tracing, environmental observation, development of a social network database, the use of peer outreach workers, provision of care, testing and treatment on the street, in addition to an outbreak response team that was flexible and could adapt rapidly to emerging issues.

Although adopting an SNA has increased the number of cases of syphilis identified and linked cases, it has also provided several challenges. Approximately 1.5 years was required to develop useful network maps. User friendly computer programs to develop those maps were initially difficult to utilise and required specially designed programs to input data from widely used data management software. There were few resources available to provide guidelines to support peer initiatives. The data set was often incomplete and took repeated contacts with cases and people of interest to gather needed information and to conduct testing. Certain individuals, regardless of motivation or interviewing methods employed, were unwilling to provide any contact information.

Although unknown confounders may impact on these results, it is important to place known potential confounders for these data in context. Despite the increased number of cases identified after SNA was adopted, these cases continued to be concentrated in the city of Vancouver, where the street nurses worked. There was not a geographic shift in the epidemic from rural British Columbia to urban Vancouver, indicating that there were not more cases in the work region

\section{Key message}

- Social networking can be used in to increase number of contacts and numbers of cases linked to index cases in a heterosexual syphilis outbreak.

of the street nurses for them to identify. A mass treatment intervention had been conducted over a year before SNA introduction, and as confirmed in mathematical modelling, the epidemic had returned to its previous level by $2001 .^{48}$ Primary care clinicians continued to be available for care for clients in the downtown eastside during this time period, with no discernible change in primary care service availability. If anything, there was greater access to primary care physicians as there was an expansion of one of the major clinics with additional clinic hours made available, thus indicating that the increased number of cases was not the result of decreased access to health care elsewhere. There was no major tourist event that could have resulted in an influx of sex trade clients. There was no seasonal variation to account for the larger number of cases. There was no increase in the number of street nurses offering service, or in the number of client encounters conducted by the street nurses over this time period.

Recent syphilis outbreaks have involved marginalised, vulnerable, and hard to reach populations and they have not responded to traditional STD control strategies and tactics. $^{79}$ The use of social networking methods implemented by an integrated team of outreach nurses and peer workers may be an important new tool to contain these outbreaks in the short term, and to eliminate syphilis in the long term. This report describes such a programme and provides preliminary quantitative evidence of its effectiveness in case finding in an ongoing outbreak. Continued evaluation is required to determine if the increased case finding and linked cases leads to a decrease in syphilis incidence rates in this population.

\section{CONTRIBUTORS}

The study concept and design was developed by MR, LK, DT, and GO; data were gathered by JT, CB, EJ, EW, JM, and HJ; data compilation and cleaning were conducted by EW and LK; analysis was conducted by GO and DT; social network mapping was conducted by LK and EW; first draft of the manuscript was prepared by GO, LK, and DT; all authors contributed to manuscript revision

\section{Authors' affiliations \\ G Ogilvie, L Knowles, D Taylor, J Tigchelaar, C Brunt, L James, J Maginley, H Jones, M L Rekart, Division of STD/AIDS Control, BC Centre for Disease Control, Vancouver, British Columbia, Canada E Wong, Surveillance and Risk Assessment Division, Health Canada, Vancouver, British Columbia, Canada}

Correspondence to: Gina Ogilvie, MD, MSc, STD/AIDS Control, BCCDC, 655 West 12th Avenue, Vancouver, BC, V5Z 4R4, Canada; gina.ogilvie@bccdc.ca

Accepted for publication 16 June 2004 


\section{REFERENCES}

1 Chen JL, Kodagoda D, Lawrence AM, et al. Rapid public health interventions in response to an outbreak of syphilis in Los Angeles. Sex Transm Dis 2004;29:277-84.

2 Williams LA, Klausner JD, Whittington WLH, et al. Elimination and reintroduction of primary and secondary syphilis. Am J Public Health 1999;89:1093-7.

3 Patrick DM, Rekart ML, Jolly A, et al. Heterosexual outbreak of infectious syphilis: epidemiological and ethnographic analysis and implications for control. Sex Transm Infect 2002;78(Suppl 1):i164-9.

4 Rekart ML, Patrick DM, Chakraborty B, et al. Targeted mass treatment for syphilis with oral azithromycin. Lancet 2003;361:313-14.
5 Rothenberg $\mathbf{R}$, Narramore J The relevance of social network concepts to sexually transmitted disease control. Sex Transm Dis 1996;23:24-9.

6 Brewer DD. Advances in methods for eliciting social networks. International Society for STD Research Ottawa, 2003 (abstract).

7 Michaud J, Ellen J, Johnson SM, et al. Responding to a community outbreak of syphils by targeting sex partner meeting location: an example of a risk-space intervention. Sex Transm Dis 2003;30:533-7.

8 Pourbohloul B, Rekart ML, Brunham RC. Impact of mass treatment on syphilis transmission: a mathematical modeling approach. Sex Transm Dis 2003:30:297-305.

9 Rothenberg R, Kimbrough L, Lewis-Hardy R, et al. Social network methods for endemic foci of syphilis: a pilot project.[comment]. Sex Transm Dis 2000;27:12-18.

\section{Clinical Evidence-Call for contributors}

Clinical Evidence is a regularly updated evidence-based journal available worldwide both as a paper version and on the internet. Clinical Evidence needs to recruit a number of new contributors. Contributors are healthcare professionals or epidemiologists with experience in evidence-based medicine and the ability to write in a concise and structured way.

Areas for which we are currently seeking authors:

- Child health: nocturnal enuresis

- Eye disorders: bacterial conjunctivitis

- Male health: prostate cancer (metastatic)

- Women's health: pre-menstrual syndrome; pyelonephritis in non-pregnant women

However, we are always looking for others, so do not let this list discourage you.

Being a contributor involves:

- Selecting from a validated, screened search (performed by in-house Information Specialists) epidemiologically sound studies for inclusion.

- Documenting your decisions about which studies to include on an inclusion and exclusion form, which we keep on file.

- Writing the text to a highly structured template (about 1500-3000 words), using evidence from the final studies chosen, within 8-10 weeks of receiving the literature search.

- Working with Clinical Evidence editors to ensure that the final text meets epidemiological and style standards.

- Updating the text every six months using any new, sound evidence that becomes available. The Clinical Evidence in-house team will conduct the searches for contributors; your task is simply to filter out high quality studies and incorporate them in the existing text.

- To expand the topic to include a new question about once every 12-18 months.

If you would like to become a contributor for Clinical Evidence or require more information about what this involves please send your contact details and a copy of your CV, clearly stating the clinical area you are interested in, to Klara Brunnhuber (kbrunnhuber@ bmigroup.com).

\section{Call for peer reviewers}

Clinical Evidence also needs to recruit a number of new peer reviewers specifically with an interest in the clinical areas stated above, and also others related to general practice. Peer reviewers are healthcare professionals or epidemiologists with experience in evidence-based medicine. As a peer reviewer you would be asked for your views on the clinical relevance, validity, and accessibility of specific topics within the journal, and their usefulness to the intended audience (international generalists and healthcare professionals, possibly with limited statistical knowledge). Topics are usually 1500-3000 words in length and we would ask you to review between 2-5 topics per year. The peer review process takes place throughout the year, and our turnaround time for each review is ideally 10-14 days.

If you are interested in becoming a peer reviewer for Clinical Evidence, please complete the peer review questionnaire at www. clinicalevidence.com or contact Klara Brunnhuber (kbrunnhuber@bmigroup.com). 